\title{
A New Approach to $G$-Normed Spaces: Functionally Generalized Normed Spaces
}

\author{
Ali Mutlu' ${ }^{1 *}$ Utku Gürdal ${ }^{2}$, Kübra Özkan ${ }^{1}$ \\ ${ }^{1}$ Manisa Celal Bayar University, Department of Mathematics, 45140, Manisa, Turkey, +90 2362013206 \\ ali.mutlu@cbu.edu.tr \\ ${ }^{2}$ Mehmet Akif Ersoy University, Department of Mathematics, Burdur, Turkey \\ *Corresponding author
}

Received: 15 October 2017

Accepted: 15 February 2018

DOI: 10.18466/cbayarfbe.407993

\begin{abstract}
In this paper, we introduce functionally generalized normed spaces as a generalization of $G$-metric spaces and normed spaces. Some constructions are described within this structure and some related results are obtained. Keywords - completion, generalized Banach space, generalized metric space.
\end{abstract}

\section{Introduction and Preliminaries}

As a result of the strong link between the theories of metric spaces and normed spaces, there has been many foundations based on metric spaces and later adapted to normed structures or vice versa. Among many others, generalized metric spaces, widely known as G-metric spaces, which were first defined by Mustafa and Sims [1] as an alternative to $D$ - metric spaces [2]; have been assumed to have connection to some normed-type structure, which would be called as generalized normed space.

So far, various generalized normed space definitions were given $[3,4]$. There are some possible different approaches to $G$-normed spaces, change the binary operation of the linear space to a special type of trinary operation, to consider a norm-like function with two variables or to give an additional algebraic operation on real numbers.

In this study we introduce generalized pseudometric spaces, then we give a definition of generalized normed space, which is based on a type of norm functional with two variables, without giving any additional algebraic structure, such that the theory of normed spaces can be embedded into the theory of generalized normed spaces and every generalized normed space will have an underlying generalized metric space structure. We also define $G$ - Banach spaces and construct product spaces, quotient spaces and completions within the new structure. 
In 2006, Mustafa and Sims introduced the notion of generalized metric space, as an alternative to the notions of 2-metric space [5] and $D$ - metric space [2]. We give an equivalent definition as follows:

Definition 1.1: [1] A function $G: X \times X \times X \rightarrow \mathbb{R}$, where $X$ is a nonempty set, is called a generalized metric, or $G$-metric, on the set $X$, if the following are satisfied for all $a, x, y, z \in X$.

(G0) $G(x, y, z)=0$ implies $x=y=z$,

(G1) $G(x, x, x)=0$,

(G2) $y \neq z$ implies $G(x, x, z) \leq G(x, y, z)$,

(G3) Symmetry in all variables, that is

$$
\begin{aligned}
& G(x, y, z)=G(x, z, y)=G(y, x, z) \\
= & G(y, z, x)=G(z, x, y)=G(z, y, x)
\end{aligned}
$$$$
\text { (G4) } G(x, y, z) \leq G(x, a, a)+G(a, y, z)
$$

A generalized metric space, or $G$ - metric space, is a pair $(X, G)$, where $G$ is a $G$-metric on $X$. A $G$-metric space $(X, G)$ with the property that $G(x, y, y)=G(y, x, x)$ for all $x, y \in X$ is called symmetric.

Definition 1.2: [1] Let $(X, G)$ be a $G$-metric space, $x_{0} \in X$ and $r>0$. Then the $G-$ ball with center $x_{0}$ and radius $r$ is defined as

$$
B\left(x_{0}, r\right):=\left\{y \in X: G\left(x_{0}, y, y\right)<r\right\}
$$

Every $G$ - metric $G$, generates a metric $d_{G}$. For consistency, we consider it, among other equivalent forms, as

$$
d_{G}(x, y)=\frac{1}{2}[G(x, y, y)+G(x, x, y)]
$$

The family of all $G$-balls in a $G$ - metric space $(X, G)$ forms a base for a topology on $X$. This topo$\log y$ is the same as the topology corresponding to the metric $d_{G}[1]$.

Formerly $G$-pseudometric spaces were defined with the name pseudo- $G-$ metric space in [6]. While the axioms $\left(G_{2}\right)$ and $\left(G_{3}\right)$ of Definition 1.1 in [6] together force the structure to be a $G$ - metric space, the purpose of the definition is clear and since $\left(G_{2}\right)$ was never used there, all results obtained were true. However for completeness, here we redefine the same concept with alternative axioms and another name.

Definition 1.3: Let $X$ be a nonempty set. A function $G: X \times X \times X \rightarrow \mathbb{R}$ satisfying the axioms (G1), (G2), (G3) and (G4) in Definition 1.1 is called a generalized pseudometric, or $G$ - pseudometric, on the set $X$. A $G$-pseudometric space is a pair $(X, G)$, where $G$ is a $G$-pseudometric on $X$.

\section{$2 G$ - normed spaces}

Let $X$ be any semigroup with the identity 0 , and $\|\cdot\|:, X \times X \rightarrow \mathbb{R}$ be any mapping. In this article, we consistently use the notation $\|\cdot\|:=\|\cdot, 0\|$, that is $\|a\|=\|a, 0\|$ for all $a \in X$.

Definition 2.1: Let $X$ be a real vector space and $\|\cdot, \cdot\|: X \times X \rightarrow \mathbb{R}$ be a mapping satisfying the following for all $a, b, c \in X$ and $\lambda \in \mathbb{R}$. 
(N1) $\|a\| \leq\|a, b\|$

(N2) $\|a+b, c\| \leq\|a\|+\|b, c\|$

(N3) $\|\lambda a\|=|\lambda| \cdot\|a\|$

(N4) $\|a, b\|=\|b, a\|$

(N5) $\|a, b\|=\|a, a-b\|$

Then $\|\cdot, \cdot\|$ is called a generalized seminorm, or $G-$ seminorm on $X$. If additionally

$$
\|a, b\|=0 \Rightarrow a=b=0
$$

is satisfied, then $\|\cdot, \cdot\|$ is called a $G-$ norm. A $G-$ (semi)normed space is a pair $(X,\|\cdot\|$,$) , where \|\cdot, \cdot\|$ is a $G$-(semi)norm on $X$.

To avoid confusion with older definitions, we also use the name "functionally generalized normed space".

Example 2.2: Let $(X,\|\cdot\|)$ be a (semi)normed space, then it is easy to show that the three mappings $\|\cdot \cdot\|_{r},\|\cdot \cdot\|_{t},\|\cdot \cdot\|_{e}: X \times X \rightarrow \mathbb{R}$ defined as

$$
\begin{aligned}
\|a, b\|_{r} & =\max \{\|a\|,\|b\|,\|a-b\|\}, \\
\|a, b\|_{t} & =\frac{1}{2}(\|a\|+\|b\|+\|a-b\|) \\
\|a, b\|_{e} & =\frac{1}{\sqrt{2}}\left(\|a\|^{2}+\|b\|^{2}+\|a-b\|^{2}\right)^{1 / 2}
\end{aligned}
$$

for all $a, b \in X$, are $G-$ (semi)norms on $X$, such that $\|\cdot\|_{r}=\|\cdot\|_{t}=\|\cdot\|_{e}=\|\cdot\|$.

Example 2.3: Let $G$ be a symmetric $G$-(pseudo)metric space on a vector space $X$, with the properties

$$
G(x+a, y+a, z+a)=G(x, y, z),
$$

for all $x, y, z, a \in X$,

$$
G(\lambda x, \lambda y, \lambda z)=|\lambda| \cdot G(x, y, z),
$$

for all $x, y, z \in X, \lambda \in \mathbb{R}$. Then

$\|\cdot, \cdot\|: X \times X \rightarrow \mathbb{R},\|a, b\|=G(a, b, 0)$

defines a $G-$ (semi)norm on $X$. In particular, to see (N2) and (N4) note that

$$
\begin{aligned}
G(a+b, c, 0) & \leq G(a+b, b, b)+G(b, c, 0) \\
& =G(a, 0,0)+G(b, c, 0)
\end{aligned}
$$

and

$G(a, b, 0)=G(0, b-a,-a)=G(0, a-b, a)$.

Example 2.4: If $p$ and $q$ are $G-$ (semi)norms on $X$, and $\lambda \in \mathbb{R}^{+}$, then $p+q$ and $\lambda p$ are $G-$ (semi)norms on $X$.

Example 2.5: In the Euclidean space $\mathbb{R}^{n}$, consider two arbitrary points $a$ and $b$. If $a, b$ and the origin define a triangle, then set $\|a, b\|_{t}$ equal to half of the perimeter of the triangle. If $a, b$ and the origin together define a line, then let $\|a, b\|_{t}$ denote the maximum length of the line segments defined by these points. And if $a$ and $b$ are equal to the origin, then suppose that $\|a, b\|_{t}=0$. Then $\|\cdot, \cdot\|_{t}$ is a $G$-norm on $\mathbb{R}^{n}$. We call this the usual-triangular $G$-norm on $\mathbb{R}^{n}$. More generally, if $p$ is any seminorm on a vector space $X$, then the $p$-triangular $G$-seminorm on $X$ is formulated as

$\|a, b\|_{t}=\frac{1}{2}(p(a)+p(b)+p(a-b))$.

In particular on $\mathbb{R}$, the usual-triangular $G$-norm is defined as 
$\|a, b\|_{t}=\frac{1}{2}(|a|+|b|+|a-b|)$,

for all $a, b \in \mathbb{R}$.

Example 2.6 : Let $p$ be a seminorm on a vector space $X$. The $p$-rectangular $G$-seminorm on $X$ is given $\|a, b\|_{r}=\max \{p(a), p(b), p(a-b)\}$.

In particular, the usual-rectangular $G$ - norm on $\mathbb{R}$ is given by $\|a, b\|_{r}=\max \{|a|,|b|,|a-b|\}$.

Example 2.7: For any given seminorm $p$, the $p$-elliptic $G-$ seminorm is defined as

$\|a, b\|_{e}=\frac{1}{\sqrt{2}}\left[p(a)^{2}+p(b)^{2}+p(a-b)^{2}\right]^{1 / 2}$.

In particular, the usual-elliptic $G$ - norm on $\mathbb{R}^{n}$ can be formulated as

$$
\|a, b\|_{e}=\sqrt{\sum_{i=1}^{n}\left(a_{i}^{2}+b_{i}^{2}-a_{i} b_{i}\right)},
$$

where $a=\left(a_{1}, \ldots, a_{n}\right), b=\left(b_{1}, \ldots, b_{n}\right) \in \mathbb{R}^{n}$ and the usual-elliptic $G-$ norm on $\mathbb{R}$ is defined as

$$
\|a, b\|_{e}=\sqrt{a^{2}+b^{2}-a b} .
$$

In follows $\mathbb{R}$ is always assumed to have the usual-elliptic $G-$ norm on it.

Definition 2.8: A $G-($ semi)norm $\|\cdot$,$\| on X$, with the following properties $\left(\mathrm{N} 2^{*}\right)$ and $\left(\mathrm{N} 3^{*}\right)$, which are stronger than (N2) and (N3), respectively, is called an equi- $G-$ (semi)norm on $X$.

$$
\left(\mathrm{N} 2^{*}\right)\|a+b, c+d\| \leq\|a, c\|+\|b, d\|,
$$

for all $a, b, c, d \in X$,

$$
\left(\mathrm{N}^{*}\right)\|\lambda a, \lambda b\| \leq|\lambda| \cdot\|a, b\| \text { for all } a, b \in X,
$$

$\lambda \in \mathbb{R}$.

Example 2.9: $G$ - (semi)normed in Examples 2.2, 2.5, 2.6 and 2.7 are always equi- $G-$ (semi)norms.

Proposition 2.10: If $(X,\|, \cdot\|)$ is a $G$-seminormed space, the following are true for all $a, b \in X$

(a) $\|0\|=0$,

(b) $\|a, a\|=\|a\|$,

(c) $\|a, b\|=\|-a,-b\|$,

(d) $\frac{1}{2}(\|a\|+\|b\|) \leq\|a, b\| \leq\|a\|+\|b\|$.

Proof:

(a) $\|0\|=\|0 a\|=|0| \cdot\|a\|=0$.

(b) $\|a, a\|=\|a, a-a\|=\|a, 0\|=\|a\|$.

(c) $\|-a,-b\|=\|-a,-a-(-b)\|=\|b-a,-a\|$

$=\|b-a, b-a-(-a)\|$ $=\|b, b-a\|=\|b, a\|=\|a, b\|$.

(d) $\frac{1}{2}(\|a\|+\|b\|) \leq \frac{1}{2}(\|a, b\|+\|a, b\|)$ $=\|a, b\|=\|a+0, b\|$ $\leq\|a\|+\|0, b\|=\|a\|+\|b\|$.

Proposition 2.11: Let $(X,\|\cdot\|$,$) be a G-$ (semi)normed space. Then $\left(X, G_{\|, \cdot\|}\right)$ is a $G-$ (pseudo)metric space on $X$, where $G_{\|\cdot,\|}: X \times X \times X \rightarrow \mathbb{R}$ is given by 
$G_{\|\cdot,\|}(x, y, z)=\|x-y, x-z\|$ for all $x, y, z \in X$. In addition, $G_{\|, \cdot\|}$ is symmetric and $G_{\|,\|, \|}(a, b, 0)=\|a, b\|$ for all $a, b \in X$.

Proof: Let $x, y, z, a \in X$

(G1) $G_{\|, \cdot\|}(x, x, x)=\|0,0\|=0$.

(G2) From (N1) and (N4) we get

$$
\begin{aligned}
G_{\|\cdot,\|}(x, x, z) & =\|0, x-z\|=\|x-z\| \\
& \leq\|x-y, x-z\|=G_{\|\cdot,\|}(x, y, z) .
\end{aligned}
$$

(G3) By (N4) we have

$$
\begin{aligned}
& G_{\|\cdot,\|}(x, y, z)=G_{\|\cdot,\|}(x, z, y), \\
& G_{\|\cdot, \cdot\|}(y, x, z)=G_{\|\cdot,\|}(y, z, x)
\end{aligned}
$$

and

$$
G_{\|\cdot,\|}(z, x, y)=G_{\|\cdot,\|}(z, y, x) .
$$

Also,

$$
\begin{aligned}
G_{\|\cdot,\|}(x, y, z) & =\|x-y, x-z\| \\
& =\|x-y,(x-y)-(x-z)\| \\
& =\|x-y, z-y\| \\
& =\|y-x, y-z\| \\
& =G_{\|\cdot, \cdot\|}(y, x, z)
\end{aligned}
$$

and

$$
\begin{aligned}
G_{\|\cdot,\|}(x, y, z) & =\|x-y, x-z\| \\
& =\|x-z, x-y\| \\
& =\|x-y, z-y\| \\
& =\|z-x, z-y\| \\
& =G_{\|\cdot,\|}(z, x, y)
\end{aligned}
$$

by Proposition 2.10 (c).

(G4) Observe that

$$
\begin{aligned}
G_{\|\cdot,\|}(x, y, z) & =\|x-y,(x-y)-(x-z)\| \\
& =\|(x-a)+(a-y), z-y\| \\
& \leq\|x-a\|+\|a-y, z-y\| \\
& =G_{\|\cdot, \cdot\|}(x, a, a)+G_{\|\cdot, \cdot\|}(a, y, z) .
\end{aligned}
$$

If, in addition $\|\cdot$,$\| is a G$-norm, then $(\mathrm{G} 0)$ is obtained by

$$
\begin{aligned}
G_{\|, \cdot\|}(x, y, z)=0 & \Rightarrow\|x-y, x-z\|=0 \\
& \Rightarrow x-y=x-z=0 \Rightarrow x=y=z .
\end{aligned}
$$

Since

$$
\begin{aligned}
G_{\|\cdot,\|}(x, y, y) & =\|x-y, x-y\| \\
& =\|y-x, y-x\| \\
& =G_{\|\cdot,\|}(y, x, x),
\end{aligned}
$$

$G_{\|\cdot,\|}$ is symmetric.

By Proposition 2.10 (c) and (G3),

$G_{\|\cdot,\|}(a, b, 0)=\|a, b\|$

for all $a, b \in X$.

Proposition 2.12 : Let $(X,\|\cdot\|$,$) be a G-($ semi)normed space. Then the induced functional $\|\cdot\|: X \rightarrow \mathbb{R}$, defined as

$$
\|a\|=\|a, 0\|
$$

for all $a \in X$, is a (semi)norm on $X$.

Proof: Follows directly from the definitions. 
Proposition 2.12 and Example 2.2 together shows that, $G-$ (semi)normed space properly generalize (semi) normed spaces.

Every $G$-seminormed space $(X,\|, \cdot\|)$ will be assumed to have the topology of the corresponding seminormed space $(X,\|\cdot\|)$. Also, since

$$
\begin{aligned}
d_{G_{\|, \cdot\|}}(x, y) & =\frac{1}{2}\left[G_{\|\cdot,\|}(x, y, y)+G_{\|\cdot,\|}(x, x, y)\right] \\
& =\frac{1}{2}[\|x-y, x-y\|+\|0, x-y\|] \\
& =\|x-y\|
\end{aligned}
$$

by Proposition 2.10 (b) and (N4), the pseudometrics, obtained by the seminorm and the $G$-pseudometric, both corresponding to a fixed $G$-seminorm, are same.

Since every $G$-seminorm $\|\cdot$,$\| defines a G$-pseudometric $G_{\|\cdot,\| \|}$ which also defines a pseudometric $d_{G_{\|\cdot\|}}$ that has an underlying topology $\tau_{d_{G \mid ; \|}}$, the theory of $G$ - seminormed spaces is only isometrically different from the theory of seminormed spaces, a similar case with the case of $G$-metric spaces and metric spaces. So, many concepts defined on topological, pseudometric, and $G-$ pseudometric spaces, are readily available on $G$-normed spaces.

Definition 2.13: Let $\left(X,\|\cdot, \cdot\|_{1}\right)$ and $\left(Y,\|\cdot, \cdot\|_{2}\right)$ be two $G$-seminormed spaces. A function $f: X \rightarrow Y$ is called $G$-continuous if and only if it is continuous with respect to the corresponding topologies.

Definition 2.14: (i) Let $(X,\|\cdot, \cdot\|)$ be a $G$-seminormed space. A sequence $\left(a_{n}\right)$ on $X$ is $G$-convergent to a point $a \in X$ if and only if it converges to the point $a$, in the corresponding topological space. In that case we write $\left(a_{n}\right) \rightarrow a, \lim _{n} a_{n}=a$ or simply $\lim a_{n}=a$.

(ii) Let $(X,\|, \cdot\|)$ be a $G$ - seminormed space. A sequence $\left(a_{n}\right)$ on $X$ is called $G$-Cauchy if and only if it is a Cauchy sequence on the pseudometric space $\left(X, d_{G_{\|;\|}}\right)$.

(iii) A $G$-seminormed space $(X,\|\cdot, \cdot\|)$ is called $G$-complete if and only if every $G$-Cauchy sequence on $X$ is $G$-convergent.

\section{$3 G$ - Banach Spaces}

Now we are in a position to define generalized Banach spaces.

Definition 3.1 : A complete $G$ - normed space is called a $G$-Banach space.

Proposition 3.2 : A $G$-normed space $(X,\|, \cdot\|)$ is a $G$ - Banach space if and only if $(X,\|\cdot\|)$ is a Banach space.

Proof: The topology induced by $\|\cdot, \cdot\|$ is equal to the topology of the norm $\|\cdot\|$ and the definitions of $G$ - convergence, $G$-Cauchy sequence and $G$-completeness on $(X,\|\cdot\|$,$) are equivalent to the definitions of conver-$ gence, Cauchy sequence and completeness on $(X,\|\cdot\|)$.

Proposition 3.3: Let $(X,\|\cdot, \cdot\|)$ be a $G$-seminormed space. Then $\|\cdot, \cdot\|$ is jointly $G$ - continuous in both variables. 
Proof: Since $\|a, b\|=G_{\|\cdot,\|}(a, b, 0)$ for all $a, b \in X$, the

proof follows from Proposition 8 in [1].

Definition 3.4 : Let $\left(X,\|\cdot, \cdot\|_{1}\right)$ and $\left(Y,\|\cdot, \cdot\|_{2}\right)$ be $G-$ seminormed spaces, A function $f: X \rightarrow Y$ is called $G$-bounded, if there exists a constant $\alpha \in \mathbb{R}$ such that $\|f(x), f(y)\|_{2} \leq \alpha \cdot\|x, y\|_{1}$

for all $x, y \in X$.

Proposition 3.5 : A function

$f:\left(X,\|\cdot, \cdot\|_{1}\right) \rightarrow\left(Y,\|\cdot, \cdot\|_{2}\right)$

between $G$-seminormed spaces is $G$-bounded if and only if it is bounded with respect to the induced seminorms $\|\cdot\|_{1}$ and $\|\cdot\|_{2}$.

Proof: If there exists constant $\alpha \in \mathbb{R}$ such that

$$
\|f(x), f(y)\|_{2} \leq \alpha \cdot\|x, y\|_{1}
$$

for all $x, y \in X$, then

$$
\begin{aligned}
\|f(x)\|_{2} & =\|f(x), f(x)\|_{2} \\
& \leq \alpha \cdot\|x, x\|_{1}=\alpha \cdot\|x\|_{1} .
\end{aligned}
$$

Conversely, assume that $f:\left(X,\|\cdot\|_{1}\right) \rightarrow\left(Y,\|\cdot\|_{2}\right)$ is bounded by a constant $\alpha$. Then $f$ is $G$-bounded, since for every $x, y \in X$,

$$
\begin{aligned}
\|f(x), f(y)\|_{2} & \leq\|f(x)\|_{2}+\|f(y)\|_{2} \\
& \leq \alpha \cdot\left(\|x\|_{1}+\|y\|_{1}\right) \leq 2 \alpha \cdot\|x, y\|_{1}
\end{aligned}
$$

by Proposition 2.10 (d).
Corollary 3.6 : Let $(X,\|\cdot\|$,$) be a G$-normed space. Then a linear functional $f$ on $X$ (that is a function

$f: X \rightarrow \mathbb{R}$ which is linear with respect to vector space structures) is $G$-bounded if and only if it is $G$-continuous.

Proposition 3.7 : Let $\left(X,\|\cdot, \cdot\|_{1}\right)$ and $\left(Y,\|\cdot, \cdot\|_{2}\right)$ be $G$-Banach spaces, and $B(X, Y)$ denote the set of all $G$-bounded linear mappings from $X$ to $Y$. Then the functions

$\|\cdot \cdot\|^{0}: X^{*} \times X^{*} \rightarrow \mathbb{R}$ and $\|\cdot \cdot \cdot\|^{\#}: X^{*} \times X^{*} \rightarrow \mathbb{R}$ defined as follows are $G$-norms on $B(X, Y)$ such that $\left(B(X, Y),\|\cdot, \cdot\|^{0}\right)$ and $\left(B(X, Y),\|\cdot \cdot\|^{\#}\right)$ be $G-$ Banach spaces.

$$
\|f, g\|^{0}=\sup \left\{\|f(x), g(x)\|_{2}:\|x\|_{1} \leq 1, x \in X\right\} .
$$

$\|f, g\|^{\#}=\sup \left\{\|f(x), g(y)\|_{2}:\|x, y\|_{1} \leq 1, x, y \in X\right\}$.

Proof: It is straightforward to show that $\|\cdot, \cdot\|^{0}$ and $\|\cdot, \cdot\|^{\#}$ are both $G-$ norms on $B(X, Y)$. Moreover noting that, by $(N 1),\|x\| \leq 1$ if and only if there exists a $y \in X$ such that $\|x, y\| \leq 1$, it is seen that $\|\cdot\|^{0}$ and $\|\cdot\|^{\#}$ are both equal to the operator norm $\|\cdot\|$ on $B(X, Y)$ defined as

$$
\|f\|=\sup \left\{\|f(x)\|_{2}:\|x\|_{1} \leq 1, x \in X\right\},
$$

which makes $B(X, Y)$ complete [7] and thus $G-$ complete with respect to $\|, \cdot\|^{0}$ and $\|\cdot, \cdot\|^{\#}$. 
Definition 3.8 : Let $(X,\|\cdot\|$,$) be a G$ - Banach space.

Then $X^{*}$ denotes the set of all $G$-continuous (or equivalently, $G$-bounded) linear functionals on $X$ and $\left(X^{*},\|\cdot, \cdot\|^{*}\right)$ is called the dual space of $X$ if $\|\cdot \cdot \cdot\|^{*}=\|\cdot \cdot \cdot\|^{0}$ and the equi-dual space of $X$ if $\|\cdot \cdot\|^{*}=\|\cdot, \cdot\|^{\#}$.

\section{Product of $G-$ Seminormed Spaces}

Proposition 4.1: Let $\left(X_{i},\|\cdot, \cdot\|_{i}\right), i=1,2, \ldots, n$, be $G-$ (semi)normed spaces, where $n \geq 2$. Consider set $X=\prod_{i=1}^{n} X_{i}$

with the structure of product of real vector spaces $X_{i}$. Then the functions $\|\cdot, \cdot\|_{0},\|\cdot, \cdot\|_{1},\|\cdot, \cdot\|_{2}: X \times X \rightarrow \mathbb{R}$, defined as

(a) $\|a, b\|_{r}=\max _{1 \leq i \leq n}\left\|a_{i}, b_{i}\right\|_{i}$

(b) $\|a, b\|_{t}=\sum_{i=1}^{n}\left\|a_{i}, b_{i}\right\|_{i}$

(c) $\|a, b\|_{e}=\left(\sum_{i=1}^{n}\left\|a_{i}, b_{i}\right\|_{i}^{2}\right)^{1 / 2}$ for all $a=\left(a_{1}, \ldots, a_{n}\right), b \in\left(b_{1}, \ldots, b_{n}\right)$ in $X$ are $G-$ (semi)norms on $X$.

Proof: Note that, in each case it is enough to prove for $n=2$. Other results follow from induction. Consider the induced $G$-pseudometrics $G_{\|;,\|_{1}}$ and $G_{\|;,\|_{2}}$. Then by Theorem 2.3 of [6], the rules
$G_{t}(x, y, z)=G_{\|; \cdot\|_{1}}\left(x_{1}, y_{1}, z_{1}\right)+G_{\|;\|_{2}}\left(x_{2}, y_{2}, z_{2}\right)$

$G_{e}(x, y, z)=\left(G_{\|\cdot\|_{1}}\left(x_{1}, y_{1}, z_{1}\right)^{2}+G_{\|; \cdot\|_{2}}\left(x_{2}, y_{2}, z_{2}\right)^{2}\right)^{1 / 2}$,

where

$x=\left(x_{1}, x_{2}\right), y=\left(y_{1}, y_{2}\right), z=\left(z_{1}, z_{2}\right) \in X_{1} \times X_{2}$, define $G$-pseudometrics on $X$. Let $G$ denote $G_{r}, G_{t}$ or $G_{e}$. Note that $G$ satisfies

$G(x+a, y+a, z+a)=G(x, y, z)$

and $G(\lambda x, \lambda y, \lambda z)=|\lambda| \cdot G(x, y, z)$, for all $x, y, z, a \in X$. So $\|\cdot \cdot\|: X \times X \rightarrow \mathbb{R}$, $\|a, b\|=G(a, b, 0)$

defines a $G$-seminorm on $X$. Also, for $i=r, t, e$ we have $G_{i}(a, b, 0)=\|a, b\|_{i}$, which proves that $\|\cdot, \cdot\|_{i}$ are $G$-seminorms on $X$. Moreover, these $G$-seminorms have the topology of the product space, since they induce the three known product space seminorms.

\section{Quotient Space of a $G$-Seminormed Space}

Let $(X,\|\cdot\|$,$) be a G-($ semi)normed space, and let $U$ be a linear subspace of $X$. Define a relation $\equiv$ on $X$ by $a \equiv b \Leftrightarrow a-b \in U$, for all $a, b \in X$. Consider the quotient set

$$
X / U=\{[a]: a \in X\} .
$$

$X / U$ is also a vector space with the operations defined as $[a]+[b]:=[a+b]$ and $\lambda[a]:=[\lambda a]$ for all $[a],[b] \in X / U$ and $\lambda \in \mathbb{R}$.

$G_{r}(x, y, z)=\max \left\{G_{\|\cdot\|_{1}}\left(x_{1}, y_{1}, z_{1}\right), G_{\|\cdot\|_{2}}\left(x_{2}, y_{2}, z_{2}\right)\right\}$ Now we give a $G-($ semi)norm on $X / U$. 
Proposition 5.1: Let $(X,\|\cdot\|$,$) be a G$-seminormed space, and let $U$ be a linear subspace of $X$. Then the function $\|\cdot, \cdot\|_{,}: X / U \times X / U \rightarrow \mathbb{R}$, defined as

$$
\|[a],[b]\|_{/}=\inf _{u, v \in U}\|a+u, b+v\|
$$

is a $G$-seminorm on $X / U$. Moreover if $\|\cdot, \cdot\|$ is a $G$-norm and the subspace $U$ is closed in $X$, then also $\|\cdot \cdot\|$, is a $G-$ norm.

Proof: (N1) Since $\|a+u, v\| \geq\|a+u, 0\|$ and $0 \in U$,

$$
\begin{aligned}
\|[a]\|_{/} & =\|[a],[0]\|_{/}=\inf _{u, v \in U}\|a+u, v\|=\inf _{u \in U}\|a+u\| \\
& \leq \inf _{u, v \in U}\|a+u, b+v\|=\|[a],[b]\|_{/} .
\end{aligned}
$$

(N2) We have $\left\{u+u^{\prime}: u, u^{\prime} \in U\right\}=U$, thus

$$
\begin{aligned}
\inf _{u, v \in U}\|a+b+u, c+v\| & =\inf _{u, u^{\prime}, v \in U}\left\|a+b+u+u^{\prime}, c+v\right\| \\
& \leq \inf _{u \in U}\|a+u\|+\inf _{u^{\prime}, v \in U}\left\|b+u^{\prime}, c+v\right\| \\
& =\|[a]\|,+\|[b],[c]\|_{/} .
\end{aligned}
$$

(N3) For $\lambda=0,\|0[a]\|_{/}=\|[0]\|_{/}=\inf _{u \in U}\|u\|=0$.

For $\lambda \neq 0$, observe that $\lambda U=\{\lambda u: u \in U\}=U$, so

$$
\begin{aligned}
\|\lambda[a]\|_{,} & =\inf _{u \in U}\|\lambda a+u\|=\inf _{v \in \lambda U}\|\lambda a+v\| \\
& =\inf _{u \in U}\|\lambda a+\lambda u\|=|\lambda| \cdot\|[a]\| .
\end{aligned}
$$

(N4) and (N5) are immediately seen.

Now we suppose that $\|\cdot\|,$,$\quad is a G-$ norm and $U$ is closed. Let $\|[a],[b]\|_{,}=0$. Then

$\inf _{u, v \in U}\|a+u, b+v\|=0$,

so for every $\varepsilon>0$ there exist $u_{\varepsilon}, v_{\varepsilon} \in U$ such that

$$
\left\|a+u_{\varepsilon}, b+v_{\varepsilon}\right\|<\frac{\varepsilon}{2} \text {. }
$$

This implies $\left\|a+u_{\varepsilon}\right\|<\frac{\varepsilon}{2}$. Then $\left(\left\|a+u_{1 / n}\right\|\right)$ is a sequence in $\mathbb{R}$ convergent to 0 . Since $\left\|u_{1 / n}-(-a)\right\| \rightarrow 0$, we have $\left(u_{1 / n}\right) \rightarrow-a$. $U$ is a closed subset of $X$, hence $0-a=-a \in U$, which gives $[a]=[0]$.

Corollary 5.2: Let $(X,\|\cdot\|$,$) be a G$ - Banach space, and let $U$ be a closed linear subspace of $X$. Then $\left(X / U,\|\cdot, \cdot\|_{,}\right)$is a $G-$ Banach space.

Proof: It follows from the fact that quotient space of a Banach space to a closed subspace is also a Banach space and the induced norm $\|\cdot\|$, of $X / U$ is equal to the quotient norm corresponding to $\|\cdot\|[7]$.

\section{$6 G$-Completion of a $G$-Normed Space}

Here we construct a $G$-completion of a $G$-normed space, which will be unique up to $G$-norm-isomorphism and defined as follows.

Definition 6.1: A function $f:\left(X,\|\cdot,\|_{1}\right) \rightarrow\left(Y,\|\cdot, \cdot\|_{2}\right)$ between $G$ - seminormed spaces is called a $G$-normisomorphism if and only if it is a linear bijection preserving the values of the $G$-seminorm, that is, a one-toone and surjective function such that

$$
f(\lambda x+y)=\lambda f(x)+f(y)
$$

and

$$
\|f(x), f(y)\|_{2}=\|x, y\|_{1}
$$

for all $\lambda \in \mathbb{R}, x, y \in X$. 
Two $G$ - seminormed spaces are said to be $G$-isomorophic, if there exists a $G$-norm-isomorphism from one to the other.

Definition 6.2: Let $\|\cdot, \cdot\|: X \times X \rightarrow \mathbb{R}$ be a $G-$ (semi)normed space and $A$ be a linear subspace of $X$.

Then also the restriction function

$\left.\|\cdot \cdot\|\right|_{A \times A}: A \times A \rightarrow \mathbb{R}$

is a $G-$ (semi)norm on $A$. Together with this (semi)norm, $A$ is called a $G$-subspace of $X$. We simply write $\|\cdot, \cdot\|$ instead of $\left.\|\cdot, \cdot\|\right|_{A \times A}$.

Definition 6.3 : A $G$-completion of a $G$-normed space $(X,\|\cdot, \cdot\|)$ is a $G-$ Banach space $\left(Y,\|, \cdot\|^{\prime}\right)$ such that $(X,\|\cdot, \cdot\|)$ is $G$-isomorphic to a $G$-subspace $\left(X_{0},\|, \cdot\|^{\prime}\right)$ of $\left(Y,\|, \cdot\|^{\prime}\right)$, such that $X_{0}$ is a dense subset of $Y$ with respect to the topology of $\|\cdot, \cdot\|^{\prime}$.

Let $(X,\|, \cdot\|)$ be a $G$-normed space. We denote by $\hat{X}$, the set of all $G$-Cauchy sequences on $X$. Define a relation $\sim$ on $\hat{X}$ with

$$
\left(a_{n}\right) \sim\left(b_{n}\right): \Leftrightarrow \lim \left\|a_{n}-b_{n}\right\|=0
$$

on $X$. Definitions of $G-$ Cauchy sequence and $G-$ convergence are essentially equivalent to definitions of Cauchy sequence and convergence definitions for induced normed space $(X,\|\cdot\|)$ and it is known from the classical theory of normed spaces, that $\sim$ is an equivalence relation on $\hat{X}$ [7].

Let $X_{*}:=\hat{X} / \sim$ be the quotient vector space with operations

$$
[a]+[b]=:\left[\left(a_{n}+b_{n}\right)\right], \lambda[a]=:\left[\left(\lambda a_{n}\right)\right]
$$

for all $\lambda \in \mathbb{R}, a=\left(a_{n}\right), b=\left(b_{n}\right) \in \hat{X}$.

Lemma 6.4: Let $(X,\|\cdot\|$,$) be a G-$ normed space. Define $\|\cdot,\|_{*}: X_{*} \times X_{*} \rightarrow \mathbb{R}$ with

$$
\left\|\left[\left(a_{n}\right)\right],\left[\left(b_{n}\right)\right]\right\|_{*}=\lim \left\|a_{n}, b_{n}\right\|,
$$

for all $\left(a_{n}\right),\left(b_{n}\right) \in \hat{X}$. Then $\|\cdot, \cdot\|_{*}$ is a norm on $X_{*}$.

Proof: First, to show that $\|\cdot, \cdot\|_{*}$ is well-defined, we note that

$$
\begin{aligned}
\left\|a_{n}, b_{n}\right\| & \leq\left\|a_{n}-a_{m}\right\|+\left\|a_{m}, b_{n}\right\| \\
& \leq\left\|a_{n}-a_{m}\right\|+\left\|b_{n}-b_{m}\right\|+\left\|a_{m}, b_{m}\right\|,
\end{aligned}
$$

hence the inequality

$$
\left\|a_{n}, b_{n}\right\|-\left\|a_{m}, b_{m}\right\| \mid \leq\left\|a_{n}-a_{m}\right\|+\left\|b_{n}-b_{m}\right\|
$$

hold. On the other hand, since $\left(a_{n}\right)$ is a Cauchy sequence on $(X,\|\cdot \cdot\|)$,

$d_{G_{\|: ;} \|}\left(a_{n}, a_{m}\right)=\frac{1}{2}\left(\left\|a_{n}-a_{m}, a_{n}-a_{m}\right\|+\left\|a_{n}-a_{m}\right\|\right)<\frac{\varepsilon}{2}$

for every given $\varepsilon>0$ and sufficiently large numbers $m$ and $n$.

In particular $\left\|a_{n}-a_{m}\right\|<\frac{\varepsilon}{2}$ and similarly $\left\|b_{n}-b_{m}\right\|<\frac{\varepsilon}{2}$ which imply that $\left(\left\|a_{n}, b_{n}\right\|\right)$ is a Cauchy sequence on $\mathbb{R}$. Thus $\lim \left\|a_{n}, b_{n}\right\|$ exists.

Let $\left(a_{n}\right),\left(a_{n}^{\prime}\right),\left(b_{n}\right),\left(b_{n}^{\prime}\right) \in \hat{X}$ such that $\left[\left(a_{n}\right)\right]=\left[\left(a_{n}^{\prime}\right)\right]$ and $\left[\left(b_{n}\right)\right]=\left[\left(b_{n}^{\prime}\right)\right]$. 
Then $\lim \left\|a_{n}-a_{n}^{\prime}\right\|=\lim \left\|b_{n}-b_{n}^{\prime}\right\|=0$, and we have

$$
\begin{aligned}
\lim \left\|a_{n}, b_{n}\right\| & =\lim \left\|\left(a_{n}-a_{n}^{\prime}\right)+a_{n}^{\prime}, b_{n}\right\| \\
& \leq \lim \left(\left\|a_{n}-a_{n}^{\prime}\right\|+\left\|a_{n}^{\prime}, b_{n}\right\|\right) \\
& =\lim \left\|a_{n}^{\prime}, b_{n}\right\|=\lim \left\|\left(b_{n}-b_{n}^{\prime}\right)+b_{n}^{\prime}, a_{n}^{\prime}\right\| \\
& \leq \lim \left\|a_{n}^{\prime}, b_{n}^{\prime}\right\| .
\end{aligned}
$$

Similarly $\lim \left\|a_{n}^{\prime}, b_{n}^{\prime}\right\| \leq \lim \left\|a_{n}, b_{n}\right\|$, and this yields

$$
\left\|\left[\left(a_{n}\right)\right],\left[\left(b_{n}\right)\right]\right\|_{*}=\left\|\left[\left(a_{n}^{\prime}\right)\right],\left[\left(b_{n}^{\prime}\right)\right]\right\|_{*} .
$$

(N0) We show that, if $\left\|\left[\left(a_{n}\right)\right],\left[\left(b_{n}\right)\right]\right\|_{*}=0$, then $\left[\left(a_{n}\right)\right]=\left[\left(b_{n}\right)\right]=[(0)]$, where $(0)$ is the sequence with the constant value 0 .

$$
\left\|\left[\left(a_{n}\right)\right],\left[\left(b_{n}\right)\right]\right\|_{*}=0
$$

implies $\lim \left\|a_{n}, b_{n}\right\|=0$. Since $\|a\| \leq\|a, b\|$,

$$
\lim \left\|a_{n}-0\right\|=\lim \left\|a_{n}\right\|=0,
$$

so that $\left(a_{n}\right) \sim(0)$ and $\left[\left(a_{n}\right)\right]=[(0)]$. Similarly $\left[\left(b_{n}\right)\right]=[(0)]$.

(N1), (N2), (N3), (N4) and (N5) follow easily.

Lemma 6.5: The space $\left(X_{*},\|\cdot, \cdot\|_{*}\right)$ is complete.

Proof: The induced norm $\|\cdot\|_{*}$ corresponding to $G-$ norm $\|\cdot, \cdot\|_{*}$, is equal to the completion norm of the $X_{*}$, which makes $X_{*}$ complete, or equivalently $G$-complete.

Now we show that the $G$-normed space $(X,\|\cdot, \cdot\|)$ can be densely embedded into a subspace of the $G$-Banach $\operatorname{space}\left(X_{*},\|\cdot, \cdot\|_{*}\right)$.
Lemma 6.6: Let $X_{0}$ be the subset of $X_{*}$, which consists of equivalence classes of constant sequences on $X$, that is $X_{0}=\{[(x)]: x \in X\}$. Then $\left(X_{0},\|\cdot, \cdot\|\right)$ is a dense $G$-subspace of $\left(X_{*},\|\cdot, \cdot\|_{*}\right)$ and

$f:(X,\|\cdot, \cdot\|) \rightarrow\left(X_{0},\|\cdot, \cdot\|_{*}\right), \quad f(a)=[(a)]$

is a $G$-norm-isomorphism.

Proof: Note that $X_{0}$ is a linear subspace of $X_{*}$, thus $\left(X_{0},\|\cdot, \cdot\|_{*}\right)$ is a $G-$ subspace of $\left(X_{*},\|\cdot, \cdot\|_{*}\right)$. On the other hand, in the Banach space $\left(X_{*},\|\cdot\|_{*}\right)$, the subspace $X_{0}$ is isometric to $X$. So it is dense in $X_{*}$. Also we see that $f$ is linear. And the observation that

$$
\begin{aligned}
\|f(a), f(b)\|_{*} & =\|[(a)],[(b)]\|_{*} \\
& =\lim \|a, b\|=\|a, b\|
\end{aligned}
$$

completes the proof.

Corollary 6.7: Every $G$ - normed space $(X,\|, \cdot\|)$ has a $G$-completion.

Next, we prove the uniqueness of $G$-completions up to $G$-norm-isomorphism.

Theorem 6.8: If $(X,\|, \cdot\|)$ is a $G$-normed space, then its $G$-completions are $G$-isomorphic.

Proof: Let $\left(Y_{1},\|, \cdot\|_{*_{1}}\right)$ and $\left(Y_{2},\|, \cdot\|_{*_{2}}\right)$ be two $G-$ completions a given $G$-normed space $(X,\|, \cdot\|)$. Let $X_{i}$ be dense subsets of $Y_{i}$ such that there exist $G-$ norm-isomorphisms $f_{i}:(X,\|\cdot\|,) \rightarrow\left(X_{i},\|\cdot, \cdot\|_{*_{i}}\right)$ for $i=1,2$. 
Since $X_{1}$ is dense in $Y_{1}$, if $a \in Y_{1}$, then there exists a sequence in $X_{1}=f_{1}(X)$, which $G$-converges to $a$. We denote this sequence as $\left(f_{1}\left(a_{n}\right)\right)$, where $a_{n} \in X_{1}$. Since it is $G$-convergent, $\left(f_{1}\left(a_{n}\right)\right)$ is a $G$-Cauchy sequence, and it is a Cauchy sequence in the induced normed space. Note that $f_{1}^{-1}$ exists and it is a $G$-normisomorphism, and so is $f_{2} \circ f_{1}^{-1}$. Every $G$-normisomorphism is an isometric isomorphism on induced normed spaces, thus it preserves Cauchy sequences. Thence $\left(f_{2}\left(a_{n}\right)\right)$ is a Cauchy sequence on the induced normed space, and $G$-Cauchy on the $G$-normed space $f_{2}(X)=X_{2} \subseteq Y_{2}$ and since $Y_{2}$ is complete, $\left(f_{2}\left(a_{n}\right)\right) G$-converges to a point in $Y_{2}$. We set this point as the value $g(a)$ of $g$ at $a$.

It is easy to verify that $g$ is a well-defined linear bijection. Also $a, b \in Y$ and $\left(f_{1}\left(a_{n}\right)\right),\left(f_{1}\left(b_{m}\right)\right)$ are sequences $G$-convergent respectively to $a$ and $b$ on $X_{1}$. So

$$
\left(f_{2}\left(a_{n}\right)\right) \rightarrow g(a) \text { and }\left(f_{2}\left(b_{m}\right)\right) \rightarrow g(b) .
$$

Since $f_{1}$ and $f_{2}$ are $G$-norm-isomorphism

$$
\begin{aligned}
\left\|a_{n}, b_{n}\right\| & =\left\|f_{1}\left(a_{n}\right), f_{1}\left(b_{m}\right)\right\|_{*_{1}} \\
& =\left\|f_{2}\left(a_{n}\right), f_{2}\left(b_{m}\right)\right\|_{*_{2}} .
\end{aligned}
$$

Since $G$-seminorms are continuous in both variables, we have

$$
\begin{aligned}
\lim _{m} \lim _{n}\left\|f_{1}\left(a_{n}\right), f_{1}\left(b_{m}\right)\right\|_{*_{1}} & =\lim _{m}\left\|a, f_{1}\left(b_{m}\right)\right\|_{*_{1}} \\
& =\|a, b\|_{*_{1}}
\end{aligned}
$$

and

$$
\begin{aligned}
\lim _{m} \lim _{n}\left\|f_{2}\left(a_{n}\right), f_{2}\left(b_{m}\right)\right\|_{*_{2}} & =\lim _{m}\left\|g(a), f_{2}\left(b_{m}\right)\right\|_{*_{2}} \\
& =\|g(a), g(b)\|_{*_{2}} .
\end{aligned}
$$

Hence we conclude that $\|a, b\|_{*_{1}}=\|g(a), g(b)\|_{*_{2}}$.

\section{References}

1. Mustafa, Z. and Sims, B.; A new approach to generalized metric spaces, Journal of Nonlinear and Convex Analysis. 2006, 7(2), 289-297.

2. Dhage, B. C.; Generalized metric space and mapping with fixed point. Bulletin of the Calcutta Mathematical Society. 1992, 84, 329-336.

3. Mohammedpour, A. and Shobe, N.; Stability of additive mappings in generalized normed space, TamsuiOxf. Jornal of Informatics and Mathematical Sciences. 2013, 29(2), 201-217.

4. Shrivastava, R., Animesh, G. and Yadava, R. N.; Some mapping on $G-$ Banach space. International Journal of Mathematical Science and Engineering Applied. 2011, 5 (IV), 245-260.

5. Gahler, S.; 2-Metrische Raume und ihre topologische struktur. (German) Mathematische Nachrichten. 1963, $26,115-148$.

6. Fernandez, J. and Malviya, N.; Pseudo- $G$ - metric spaces and pseudo- $G-$ metric product spaces. South Asian Journal of Mathematics. 2013, 3 (1), 339-343.

7. Maddox, I. J.; Elements of functional analysis. Second edition. Cambridge University Press, Cambridge, 1988; pp. 242 